\title{
Presentation of Automated Procedural Guidance in Surgical Simulation: Results of Two Randomised Control Trials
}

Sudanthi Wijewickrema (BEng(Hons), PhD) ${ }^{1}$, Yun Zhou (BSc, MSc, PhD) $)^{1}$, Ioanna Ioannou (BEng(Hons)/BCom $)^{1}$, Bridget Copson ${ }^{1}$ (ABC), Patorn Piromchai (MD, MSc, FRCOT, FICS) ${ }^{1,2}$, Chenjie Yu (MD) ${ }^{3}$, Robert Briggs (MBBS, FRACS $)^{1}$, James Bailey (BEng, PhD) $)^{4}$, Gregor Kennedy (BA(Hons), PhD) ${ }^{5}$, and Stephen O’Leary (MBBS, BMedS, PhD, FRACS) ${ }^{1}$

1. Department of Surgery (Otolaryngology), University of Melbourne, Australia

2. Department of Otorhinolaryngology, Khon Kaen University, Thailand

3. Department of Otolaryngology, Nanjing University, China

4. Department of Computing and Information Systems, University of Melbourne, Australia

5. Melbourne Centre for the Study of Higher Education, University of Melbourne, Australia

\section{Corresponding author:}

Sudanthi Wijewickrema

Department of Surgery (Otolaryngology), University of Melbourne,

Level 5, Royal Victorian Eye and Ear Hospital,

32, Gisborne Street, East Melbourne, VIC 3002,

Australia

Tel - +61399298365

Fax - +61 396631958

Email-swijewickrem@unimelb.edu.au

\section{Presentations}

The content of this paper was presented at the 14th Australasian Auditory Neuroscience Workshop at Hobart, Australia on the $4^{\text {th }}$ of December 2016.

\section{Acknowledgements}


This work was funded by an Australian Research Council Linkage Project Grant, in which Cochlear Ltd. is a partner. The Biostatistics Hub at the Eastern Hill/St Vincent's Academic Centre (University of Melbourne) was consulted for statistical advice.

\begin{abstract}
Objective: To investigate the effectiveness and usability of automated procedural guidance during virtual temporal bone surgery.

Methods: Two randomised controlled trials of medical students evaluated the effectiveness of two presentation modalities of automated real-time procedural guidance in virtual reality simulation: full and step-by-step visual presentation of drillable areas. Effectiveness of each presentation modality was determined through a comparison of dissection quality of participants, evaluated by a blinded otologist, using a validated assessment scale.
\end{abstract}

Setting: University of Melbourne virtual reality temporal bone surgery simulation.

Results: While the provision of automated guidance on procedure improved performance (full: $\mathrm{p}=0.03$, step-by-step: $\mathrm{p}<0.001$ ), usage of the two different presentation modalities was vastly different (full: $3.73 \%$, step-by-step: $60.40 \%$ ).

Conclusion: Automated procedural guidance in virtual temporal bone surgery is effective in improving trainee performance. Step-by-step presentation of procedural guidance was engaging, and therefore more likely to be used by the participants.

\title{
Summary:
}

- Virtual reality (VR) simulation based surgical training still requires the presence of expert trainers as most simulators only provide limited or no automated guidance during training.

- Two different methods of presenting automated procedural guidance for temporal bone surgery were explored: display of all drillable areas and step-by-step display of regions.

- Both presentation modalities led to improved performance.

- Step-by-step display of procedural guidance was found to be more engaging and therefore useful when teaching a surgical procedure, presumably due to its interactive 
nature.

\section{Keywords}

Virtual Reality Temporal Bone Surgery, Automated Guidance in Surgery Simulation, Simulation-Based Surgical Training, Procedural Guidance 


\section{Introduction}

Surgical education has long relied on the model of apprenticeship, where an expert surgeon guides trainees during cadaveric dissection and later during surgery. But the exclusive use of apprenticeship is becoming impractical in the current climate, where there are increasing demands on surgeons' time, legislation has limited trainees' working hours and cadaveric material is less available. Virtual reality (VR) simulation is one of the more promising new approaches to surgical training because it offers an environment in which repeated, risk-free practice can be undertaken and trainees can be exposed to a wide range of cases, including rare pathologies $^{1,2}$.

At this point in time simulation based training still requires the presence of expert trainers because most simulators provide limited or no automated guidance during training. This limits the scope and flexibility of simulation in training, so we and others have begun to explore the provision of automated guidance in simulated environments. For example, Rhienmora et al. ${ }^{3}$ provided real-time feedback on individual performance metrics such as force, position, and orientation in a dental simulator by making comparisons with average metric values obtained from expert performances. Fried et al. ${ }^{4}$ quantitatively defined a range of errors for surgical performance (violation of tissue, violation of instrument tolerances, force patterns, etc.) and provided feedback in an endoscopic sinus surgery simulator by making comparisons with metrics from pre-recorded performances. The metrics used were able to differentiate an expert performance from a novice and found improvement in performance of a novice with repetition. Zhou et al. ${ }^{5,6}$ provided guidance that emulated the advice of a human expert by developing multivariate models of expert and trainee behaviour and comparing real-time drilling technique to these models. These metrics were used successfully to provide feedback on surgical technique.

Most automated real-time guidance in surgical simulation has so far focused on surgical technique (based on motor skills) and less attention has been paid to how a procedure can be taught in such an environment. We have found that the provision of automated feedback on technical aspects of surgery does not necessarily teach trainees how to perform an operation ${ }^{7}$. This suggests that additional guidance, perhaps pertaining to the sequence of steps undertaken by the surgeon, may be needed. This we refer to procedural skills training, and here we investigate 
its importance and how it relates to automated guidance on surgical technique.

Procedural advice has typically been provided to students prior to training on a simulator in the form of instructions (e.g. video tutorials) ${ }^{8-10}$. However, for trainees who are not familiar with the procedure, this may not be sufficient, and additional guidance during the procedure may be beneficial. Literature suggests 'path following', where visual cues guide the trainee on the steps pertaining to a surgical procedure, as a way to overcome this issue ${ }^{11,12}$. Passmore et al. ${ }^{13}$ provided such visual guidance using a spline that indicated the path to follow in a virtual laparoscopic simulator. Botden et al. ${ }^{14}$ presented visual cues such as arrows to guide trainees performing a suturing task. In this paper, we investigate ways of implementing procedural guidance using such visual cues within the context of temporal bone surgery simulation. We explore the effectiveness and usefulness of these forms of presentation, when provided in parallel with surgical technique guidance, through randomised controlled trials.

\section{Methods}

\subsection{Simulation Platform}

The simulation platform used in this study is a VR temporal bone surgery simulator (see Figure 1). It presents the surgeon with two slightly offset images to produce the illusion of a $3 \mathrm{D}$ operating space, when viewed through 3D glasses. Major anatomical structures that must be identified without injury during surgery, such as the facial nerve, sigmoid sinus, dura, ossicles and the labyrinth, are represented in the virtual temporal bone. The surgeon interacts with the virtual temporal bone using a pen-like haptic device (surgical drill) that provides force feedback in three dimensions.

The simulator saves a stream of data (such as drill position, force, speed, proximity to anatomical structures etc.) at regular intervals. Surgical technique feedback was provided by identifying expert and trainee behaviour patterns in this data based on existing algorithm ${ }^{5,6,7}$. 


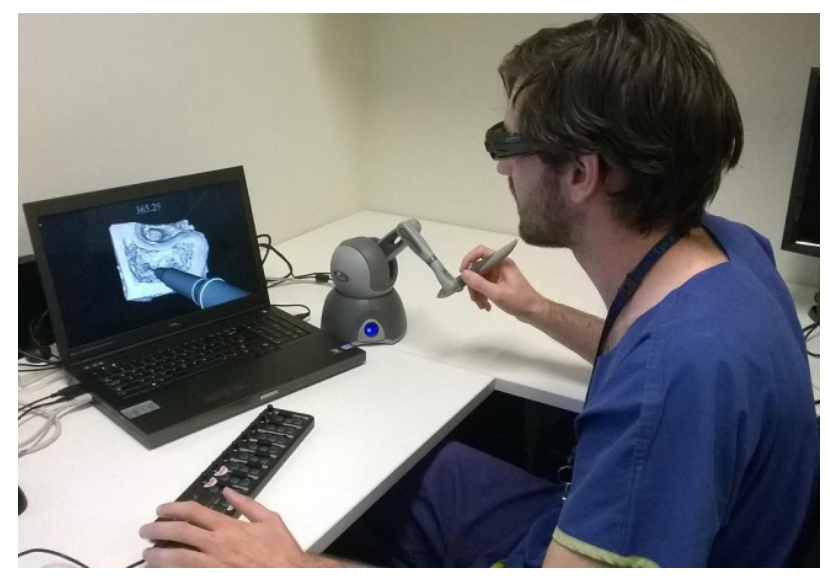

Figure 1: A surgeon performing temporal bone surgery on the temporal bone simulator

To provide procedural guidance during surgery, the performance of an expert otologist was used as a reference. The areas of the virtual temporal bone drilled by the expert were highlighted during the procedure. The areas drilled by the expert were presented in two different ways, and each was tested in a randomised controlled trial (RCT). In the first presentation method, the complete area drilled by the expert surgeon was highlighted all at the one time. In the second, the expert procedure was manually segmented into a sequence of logical steps and shown to the trainee one at a time, following the surgical flow of the operation. See Figure 2 for examples of the two presentation modalities.

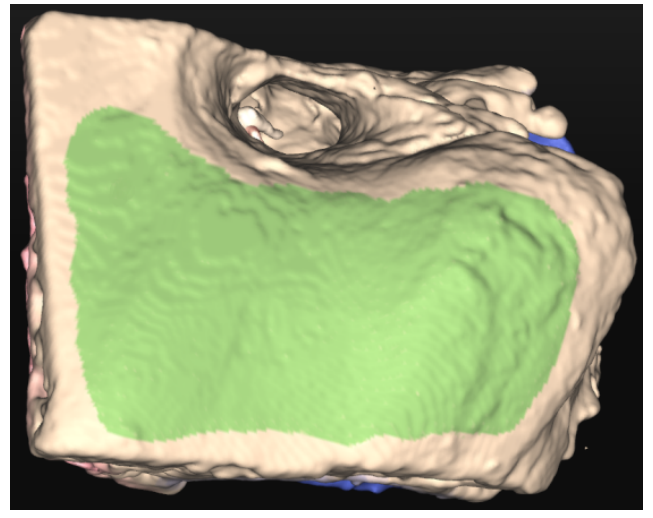

(a)

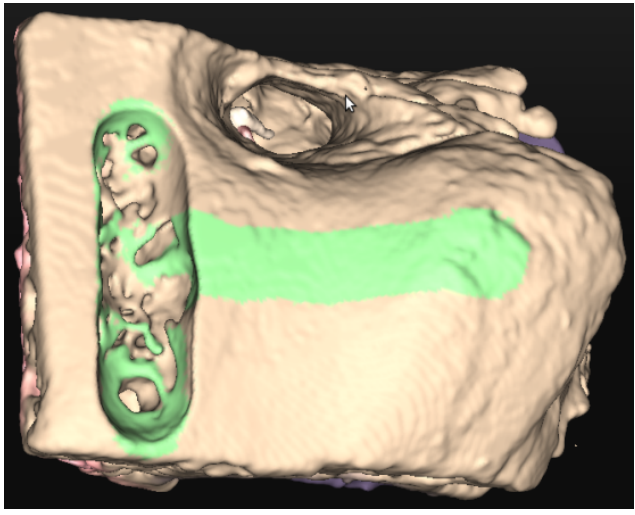

(b)

Figure 2: Procedural guidance provided in the form of coloured overlays on top of the virtual temporal bone: (a) all drillable areas highlighted at once, and (b) drillable areas highlighted once $75 \%$ of the current step has been completed. 


\subsection{Study Design}

The studies discussed in this paper were approved by a Human Research Ethics Committee of the University of Melbourne (HREC \#1135497).

First, we tested whether procedural guidance where all drillable areas were highlighted at once (Figure 2a) was effective in improving surgical performance. This involved a RCT of thirty medical students (Trial 1, Figure 3a). Participants were shown a video tutorial on how to perform a cortical mastoidectomy, taught how to use the simulator, and given 5 minutes to familiarise themselves with the VR environment. They were then stratified based on year of study and randomly allocated into one of 3 groups: 1) $\mathrm{N}$ - the 'control' group who did not receive automated guidance during drilling, 2) $\mathrm{T}$ - the 'technique guidance' group who received only feedback on surgical technique, and 3) PT - 'technique and procedural guidance' group who received guidance on where to drill as well as feedback on surgical technique. The participants then performed the virtual mastoidectomy procedure twice. To test the usability of the automated procedural guidance, those in the PT group were given the option to turn the procedural feedback on or off, as desired. We reasoned that if the participants found the procedural guidance to be useful and engaging that they would use it when given the option.

A second implementation of the procedural feedback was developed to be more interactive (Figure 2b), and this was tested in the second RCT (Trial 2, Figure 3b). This feedback provided step-by-step guidance, where the trainee was shown each step of the procedure once $75 \%$ of the previous step has been completed. This implementation was tested with a group of twenty participating medical students. The design of this study was similar to that of Trial 1 except in Trial 2, there was no group that only received technique feedback because of a lack of effect encountered in Trial 1, as presented in the results below. As in Trial 1, the usefulness of the automated guidance method was evaluated by providing the time that participants used it when given the option during the second run through the simulation. 


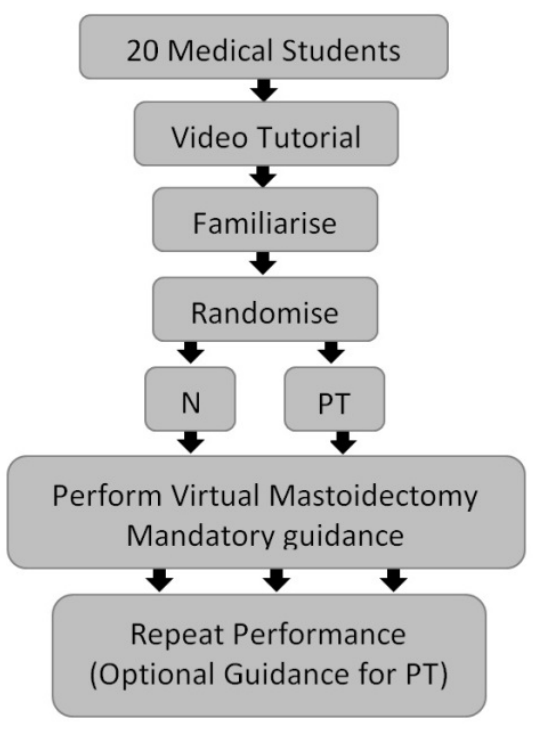

(a)

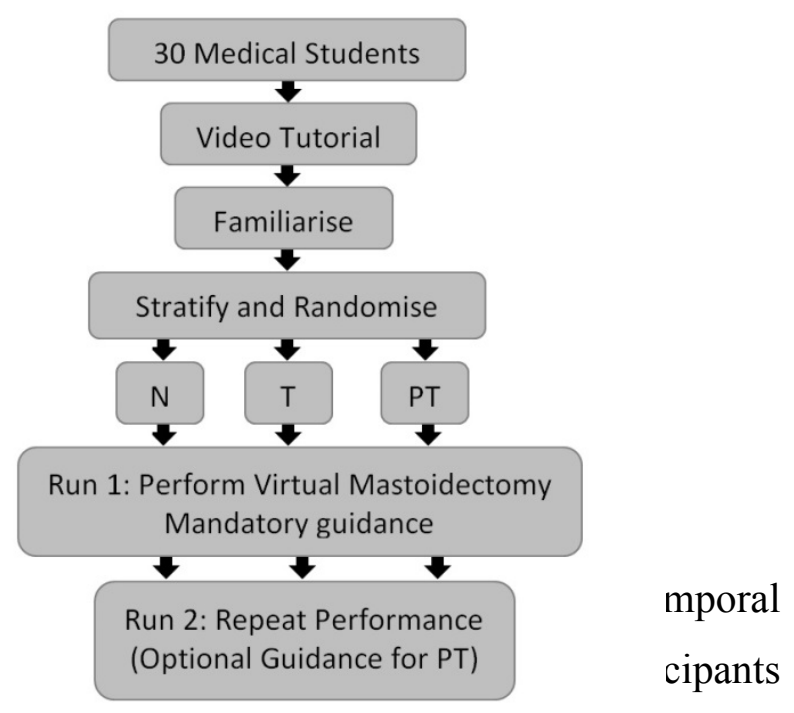

\subsection{Evaluation Method}

To evaluate the effectiveness of procedural gu bone dissection from participants that received $]$ (Optional Guidance for PT)

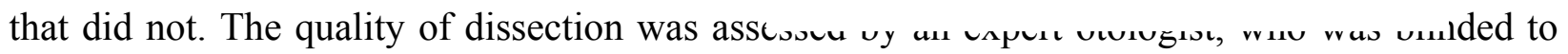
the identity of the participants and the study groups. This assessment utilised a validated tool, the Welling scale ${ }^{15}$ which is a 35 -item binary $(0,1)$ grading instrument that evaluates different aspects of dissection, such as the adequacy of saucerisation and skeletonisation.

Data from a previous study ${ }^{7}$ informed the sample size calculations. That study had 12 participants per arm, and a mean difference of $21.25 \%$ in the outcome measure was found between the feedback and no feedback groups. The standard deviation for the groups varied from $13.43 \%$ to $15.19 \%$. Assuming the worst-case scenario of a standard deviation of $15.19 \%, \mathrm{p}<0.05$, and power $=0.8$, a difference between the groups of $20.2 \%$ could be detected for 10 participants per arm (t-test, Minitab v17). Assuming that similar effects could be observed for quality of 
dissection scores, with respect to the 35 -point Welling scale ${ }^{15}$, this translates to the studies being able to detect effects (differences between the groups) of 7.4 points.

Because the experimental conditions changed between Run 1 and 2 (in the PT group guidance was turned on throughout the procedure in Run 1, while participants were given the option to turn it on when needed in Run 2), the quality of dissection scores from each run were analyzed separately. The Kruskal-Wallis statistic used because these data were not parametric. Post-hoc analyses were conducted using a Bonferroni adjustment. Differences in the quality of dissection scores between runs within each study group were evaluated using Wilcoxon's signed-rank test. The percentage of time the students used procedural guidance was calculated and compared to the total time of each procedure to evaluate the usefulness of the presentation method. A confidence interval of $95 \%$ was considered when testing for significance $(p \leq 0.05)$. Data analysis was performed using Matlab R2014a.

\section{Results}

In Trial 1, all of the areas to be drilled were highlighted simultaneously (Figure 2a). During the Run 1, use of the procedural guidance was mandated, and there was a significant difference between the end-product scores of the groups $\left(\chi^{2}(2)=6.83, p=0.03\right)$. A post-hoc analysis identified that participants who received technique and procedural feedback (PT) performed the surgery significantly better than those who received only technique feedback (T) (Figure 4). During Run 2, use of the procedural feedback was optional, and could be toggled on or off by the participant. Optional usage of the automated guidance was observed to be extremely low. Only 4 out of 10 students did not use the procedural guidance at all, and those who did, used it for $3.73 \%$ of the total time. There was no significant difference in the performance between the users and non-users of the feedback in Run 2. 


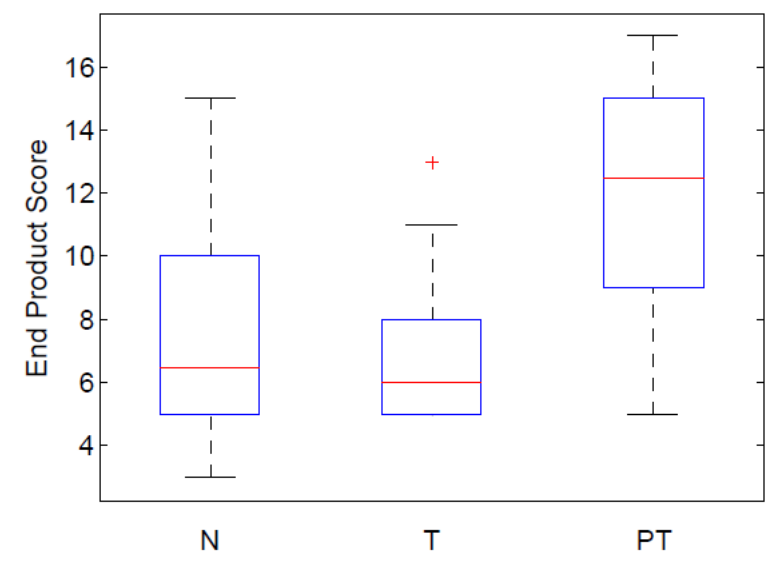

Figure 4: Quality of dissection scores of the groups for Run 1 in Trial 1. The group that received procedural guidance (PT) performed significantly better than the group that received only technique guidance (T). No significant difference in the results was observed in Run 2 where procedural guidance was optional, and was only used for approximately $4 \%$ of the time.

The quality of dissection scores of participants between runs were compared for the 3 groups. The group that received no feedback $(\mathrm{N})$ showed a significant improvement in the quality of dissection score (Wilcoxon signed-rank test, $\mathrm{p}=0.03$ ). The quality of dissection scores of $\mathrm{T}$ and PT groups did not improve significantly between runs.

We surmised that although the procedural feedback delivered in Trial 1 had been effective in improving performance, it had not engaged the user given the low rate of voluntary usage. In light of this, we devised the step-by-step guidance approach (Figure 2b), and then designed a second study (Trail 2) to test its effectiveness and acceptance. We reasoned that the most efficient experimental control would be to compare both procedural and technique feedback (PT) against no feedback, given that (a) PT had proven to be better than T alone in Trial 1, and (b) in practice both procedural and technique feedback would be provided together, so it was best to compare this with the no-feedback condition. In this experiment, all 10 students used procedural guidance during Run 2, and for an average of $60.40 \%$ of the total time. The group that received automated guidance (PT) performed significantly better with respect to the quality of dissection score when compared to the group that received no guidance $(N)$ in both runs $\left(\chi^{2}(1)=14.16, p<\right.$ 0.001 in Run 1 and $\chi^{2}(1)=9.35, \mathrm{p}=0.002$ in Run 2; Figure 5). 


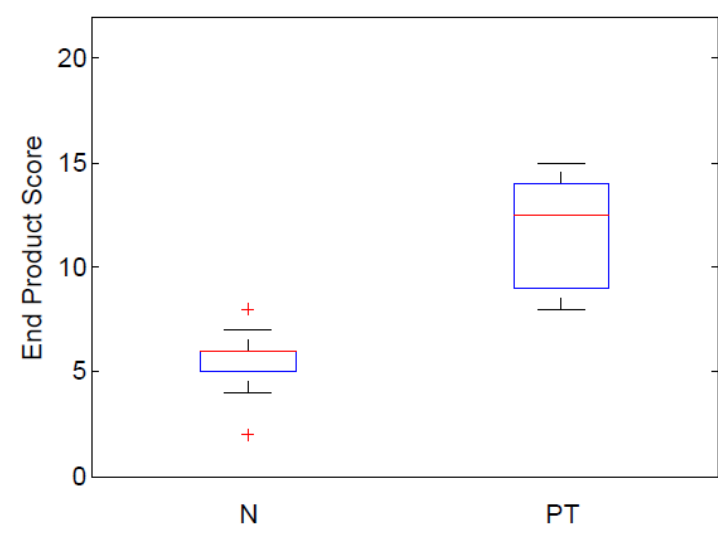

(a)

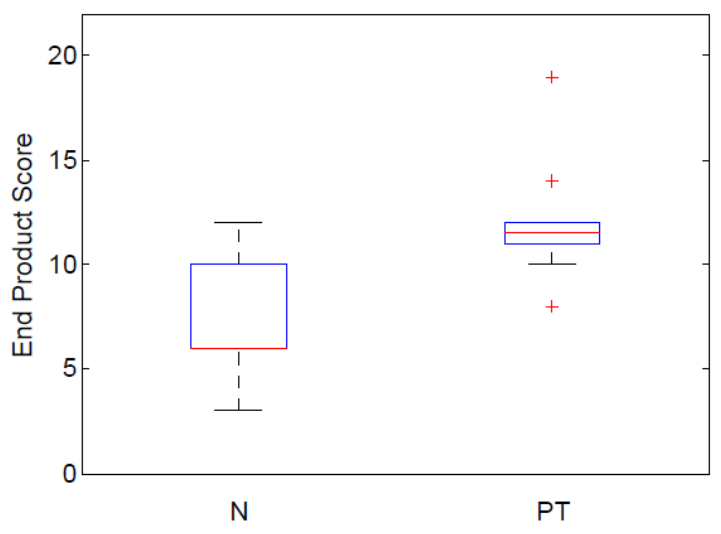

(b)

Figure 5: Quality of dissection scores of the groups in Runs 1(a) and 2(b) for trial 2. Significantly higher scores were observed in the group that received procedural guidance (PT) when compared to the group that did not (N), in both runs.

The quality of dissection scores of participants who did not receive guidance $(\mathrm{N})$ was also found to improve between the two runs (Wilcoxon signed-rank test, $p=0.03$ ). No significant difference was observed between runs in the PT group.

\section{Discussion}

The validity of a surgical simulation measures whether it is actually teaching or evaluating what was intended ${ }^{16}$. Validity is multi-faceted and includes measures such as face validity (realism of the simulator), content validity (appropriateness of the simulator to teach the subject matter), construct validity (ability to distinguish the experienced and inexperienced surgeons), and criterion validity (the effectiveness of the simulator when compared to an alternative technique, e.g. conventional training). In this paper, we discussed a method of objective criterion validity to investigate the effectiveness of the automated guidance system in teaching surgical procedures. The study design used here is similar to that used in existing studies, specifically those that evaluated guidance/feedback techniques. For example, Wijewickrema at $\mathrm{al}^{7}$ conducted a randomized controlled trial of medical students to evaluate the effectiveness of automated technical feedback. Strandbygaard et al. ${ }^{17}$ investigated the impact of instructor feedback versus 
no instructor feedback when training a complex operational task on a laparoscopic virtual reality simulator using a ransomised trial. Similarly, Kruglikova et al. ${ }^{18}$ conducted a randomized controlled trial to assess the impact of external feedback on the learning curves on a VR colonoscopy simulator.

The results of this study indicate that real-time procedural guidance was effective in improving the quality of dissection scores of participants. Both presentation methods of procedural guidance tested significantly improved the performance of trainees as compared to the control group. However, when the use of procedural feedback was optional, the step-by-step guidance method was used more often, and for longer, than the first method trialed (10 out of 10 students, $60.40 \%$ of the total time on average, compared with 4 out of 10 students, using it for only $3.73 \%$ of the total time). This suggests that the interactive step-by-step guidance was found to be more useful by the trainees. Furthermore, trainees receiving the stepwise guidance maintained their performance advantage over controls when given the option to use it (Fig 5). These findings suggests that when providing feedback, the presentation modality should be considered carefully. The more interactive and relevant to the task at hand the presentation method, the higher the level of engagement of the trainees in the procedure.

Previous literature suggested that the provision of real-time procedural feedback would not necessarily lead to improved surgical performance. In a systematic review on the effect of feedback in acquiring motor skills, Hatala et al. ${ }^{8}$ cautioned that in several surgical simulations (e.g. knot-tying ${ }^{19}$, colonoscopy ${ }^{20}$, and joint mobilisation ${ }^{21}$ ), the provision of concurrent (immediate) advice may lead to over-reliance on this feedback. The latter could result in a decrement in performance over the long-term ${ }^{22}$, as suggested by Salmoni et al. ${ }^{23}$ and Wulf \& Shea ${ }^{22}$. The improved performance seen in the present study shows this may not to be the case when feedback is applied in an engaging manner. However, further research will be required to determine how the provision of this feedback influences the retention or transfer of skills.

In order to understand why participants may have found the step-by-step feedback more engaging, it is helpful to reflect upon the nature of the surgery. Drilling of a temporal bone involves the sequential exposure of a series of anatomical structures. The step-by-step method 
followed this sequence, 'showing the way' for the trainee. In contrast, the first method presented all the bone to be drilled in one block. So, it could be argued that there was in fact more information provided in the second implementation; not only did this method provide information on where the surgeon shown drill, but also when. The improved usage and surgical outcomes with this approach is indirect evidence that these characteristics are both valued and constructive for surgeons in training. This method also lends itself to implementation in simulation with more complex anatomical variations where a step-by-step manner of guidance may aid the learner to understand a logical approach to variations in anatomy.

This study provided not just one, but two forms of concurrent real-time feedback. A previous $\operatorname{study}^{7}$, it was demonstrated that drill-handling technique of inexperienced surgeons could be driven towards that exhibited by experts by the provision of real-time technique feedback. That feedback was provided as a series of oral 'suggestions', and was optimised to be helpful but not obtrusive. The provision of both procedural and technique feedback in this study raised some interesting questions concerning implementation. Cognitive load theory suggests that the working memory used in learning is limited and as such, only a certain amount of information can be processed simultaneously ${ }^{24,25}$. It was not clear in advance that the participants would be able to assimilate the simultaneous provision of both types of feedback. To minimise this risk, we chose to present the technique and the procedural feedback through different sensory modalities. Technique feedback was provided through verbal instruction and procedural guidance was implemented as visual cues in order to be as unobtrusive as possible. This approach would appear to have been effective.

In both the studies discussed here, a significant learning effect was observed between runs in the group that received no feedback $(\mathrm{N})$. This is consistent with the predictions of the theory of deliberate practice, which states that in order for a novice to become an expert, an individual is required to undertake tasks with the explicit intent of improving' skills ${ }^{26}$. It calls for the trainee to focus on a particular task in order to improve specific aspects of performance, and involves repeated practice with coaching and immediate feedback on performance ${ }^{27}$. It is interesting however, that the participants receiving feedback (PT) did not show a statistically significant learning effect from one repetition to the next, even though their scores were better than those 
achieved by non-feedback controls. This might be due to the divided attention caused by students having to focus on multiple aspects of the procedure at once.

A limitation of this study was that there was no testing for how multiple types of feedback can affect the performance of the students, and further studies are needed to resolve this question. Further, given this was a pilot study to evaluate the usability of two presentation modes for automated feedback, there was no baseline assessment of participant performance or testing for skill retention. These would be incorporated into future studies with the ultimate goal of developing a fully automated surgical training module to teach temporal bone surgery. Initial work towards this goal, to automate the segmentation of surgical trajectory in providing procedural guidance, is discussed in Wijewickrema et $\mathrm{al}^{28}$.

In conclusion, the results of the studies discussed here indicate that real-time guidance on procedure in simulated temporal bone surgery is effective in teaching the steps of a surgical procedure. Such guidance when coupled with other forms of feedback, such as that on surgical technique, can be used to reduce the burden placed on human experts during training and pave the way for the development of fully self-directed platforms for training surgeons. 


\section{Compliance with Ethical Standards}

Funding: This research was funded through a Linkage Grant by the Australian Research Council in which Cochlear Ltd was the industry partner (Grant Number: LP130100806).

Conflict of Interest: The authors declare that they have no conflict of interest. It should be noted that authors F, G, H, and I were the recipients of the above grant (LP130100806).

Ethical approval: All procedures performed in studies involving human participants were in accordance with the ethical standards of the institutional and/or national research committee and with the 1964 Helsinki declaration and its later amendments or comparable ethical standards.

Informed consent: Informed consent was obtained from all individual participants included in the study. 


\section{References}

1 Hammoud MM, Nuthalapaty FS, Goepfert AR, Casey PM, Emmons S, Espey EL, et al. To the point: medical education review of the role of simulators in surgical training. Am J Obstet Gynecol. 2008; 199:338-43

2 Reznick RK, MacRae H. Teaching surgical skills--changes in the wind. Med Educ. 2006; 355:2664-9 3 Rhienmora P, Haddawy P, Suebnukarn S, Dailey MN. Intelligent dental training simulator with objective skill assessment and feedback. AIM. 2011; 52:115-21

4 Fried MP, Satava R, Weghorst S, Gallagher AG, Sasaki C, Ross D, et al. Identifying and reducing errors with surgical simulation. Qual Saf Health Care. 2004; 13:i19

5 Zhou Y, Bailey J, loannou I, Wijewickrema S, Kennedy G, O'Leary S. Constructive Real Time Feedback for a Temporal Bone Simulator. MICCAI. 2013. 315-22

6 Zhou Y, Bailey J, loannou I, Wijewickrema SN, O'Leary S, Kennedy G. Pattern-based real-time feedback for a temporal bone simulator. VRST. 2013. 7-16

7 Wijewickrema S, Piromchai P, Zhou Y, loannou I, Bailey J, Kennedy G, et al. Developing effective automated feedback in temporal bone surgery simulation. Otolaryngol Head Neck Surg. 2015; 152:10828

8 Hatala R, Cook DA, Zendejas B, Hamstra SJ, Brydges R. Feedback for simulation-based procedural skills training: a meta-analysis and critical narrative synthesis. Adv Health Sci Educ. 2014; 19:251-72

9 Stefanidis D, Korndorffer JR, Heniford BT, Scott DJ. Limited feedback and video tutorials optimize learning and resource utilization during laparoscopic simulator training. Surgery. 2007; 142:202-6

10 Scott DJ, Goova MT, Tesfay ST. A cost-effective proficiency-based knot-tying and suturing curriculum for residency programs. J Surg Res. 2007; 141:7-15

11 Lamata P, Enrique J, Bello F, Kneebone RL, Aggarwal R. Conceptual framework for laparoscopic VR simulators. IEEE Comput Graph Appl. 2006; 69-79

12 Crossan A, Brewster S, Reid S, Mellor D. Multimodal feedback cues to aid veterinary training simulations. HHCl. 2000; 45-9

13 Passmore PJ, Nielsen CF, Cosh W, Darzi A. Effects of viewing and orientation on path following in a medical teleoperation environment. IEEEVR. 2001; 209-15

14 Botden SM, de Hingh IH, Jakimowicz JJ. Meaningful assessment method for laparoscopic suturing training in augmented reality. Surg endosc. 2009; 23:2221-8

15 Butler NN, Wiet GJ. Reliability of the Welling scale (WS1) for rating temporal bone dissection performance. Laryngoscope. 2007; 117:1803-8

16 McDougall EM. Validation of surgical simulators. J endouro/ 2007; $21: 244-7$

17 Strandbygaard J, Bjerrum F, Maagaard M, Winkel P, Larsen CR, Ringsted C, et al. Instructor feedback versus no instructor feedback on performance in a laparoscopic virtual reality simulator: a randomized trial. Ann Surg. 2013; 257:839-44

18 Kruglikova I, Grantcharov TP, Drewes AM, Funch-Jensen P. The impact of constructive feedback on training in gastrointestinal endoscopy using high-fidelity Virtual-Reality simulation: a randomised controlled trial. Gut. 2010; 59:181-5

19 Xeroulis GJ, Park J, Moulton C-A, Reznick RK, LeBlanc V, Dubrowski A. Teaching suturing and knottying skills to medical students: a randomized controlled study comparing computer-based video instruction and (concurrent and summary) expert feedback. Surgery. 2007; 141:442-9

20 Walsh CM, Ling SC, Wang CS, Carnahan H. Concurrent versus terminal feedback: it may be better to wait. Acad Med. 2009; 84:S54-7 
21 Chang J-Y, Chang G-L, Chien C-JC, Chung K-C, Hsu A-T. Effectiveness of two forms of feedback on training of a joint mobilization skill by using a joint translation simulator. Phys Ther. 2007; 87:418-30

22 Wulf G, Shea $\mathrm{CH}$. Understanding the role of augmented feedback. Skill acquisition in sport: Research, theory and practice. 2004; 121-44

23 Salmoni AW, Schmidt RA, Walter CB. Knowledge of results and motor learning: a review and critical reappraisal. Psych bulletin. 1984; 95:355

24 Sweller J. Cognitive load during problem solving: Effects on learning. Cogn sci. 1988; 12:257-85

25 Kirschner PA. Cognitive load theory: Implications of cognitive load theory on the design of learning. Learn instr. 2002; 12:1-10

26 Ericsson KA. Deliberate Practice and the Acquisition and Maintenance of Expert Performance in Medicine and Related Domains. Acad Med. 2004; 79:S70-81

27 Cox M, Irby DM, Reznick RK, MacRae H. Teaching surgical skills-changes in the wind. $N$ Engl $J$ Med. 2006; 355:2664-9

28 Wijewickrema S, Zhou Y, Bailey J, Kennedy G, O'Leary S. Provision of automated step-by-step procedural guidance in virtual reality surgery simulation. VRST. 2016; 69-72 